\title{
Effect of many weak side modes on relative intensity noise of distributed feedback semiconductor lasers
}

\author{
Eva Peral, William K. Marshall, Dan Provenzano, and Amnon Yariv \\ Department of Applied Physics, California Institute of Technology, Pasadena, California 91125
}

(Received 4 November 1997; accepted for publication 24 December 1997)

\begin{abstract}
An increase of the relative intensity noise of nearly single-mode distributed feedback lasers with respect to that predicted by single-mode theory after propagation in dispersive fiber at frequencies up to $5 \mathrm{Ghz}$ has been measured. A simplified multimode theory is presented which explains the increase in noise. (C) 1998 American Institute of Physics. [S0003-6951(98)03508-6]
\end{abstract}

The performance of a lightwave communication system is often affected by the laser's relative intensity noise (RIN). When the link includes propagation in dispersive optical fiber, it is important to determine the RIN at the fiber output (i.e., at the receiver), which can be different from the RIN at the laser due to FM-to-AM conversion.

In addition to effects, which can be described by a single-mode laser theory, ${ }^{1}$ the side modes in a nearly singlemode laser contribute to RIN. After propagation in dispersive fiber, mode partition noise (MPN) can be converted to laser excess noise, and hence, increased RIN at the receiver. ${ }^{2}$ This is one of the factors that lead to the choice of nearly single-mode DFB lasers in communication applications.

We report here an effect of MPN that affects RIN in low-noise lasers even when the side-mode suppression ratio (SMSR) is greater than $35 \mathrm{~dB}$. Since the gain spectrum in semiconductor lasers is broad, there usually exists a broad spectrum of weak Fabry-Perot (FP) side modes. These modes, while greatly suppressed relative to the main mode, together can contribute enough power to affect the RIN in some circumstances. We demonstrate this effect experimentally, give a theory that accounts well for the observed phenomenon, and describe a set of conditions for the laser and fiber that lead to an observably increased RIN.

The carrier density fluctuations $\Delta N$ and photon density and phase fluctuations $\Delta P_{i}$ and $\Delta \phi_{i}$ at each mode $i$, at modulation frequency $\Omega$ can be obtained by using smallsignal rate equations. Nonlinear phenomena such as spectra and spatial hole burning are included here phenomenologically by using an expression for the gain, which includes dependence on the photon density of each cavity mode,

$$
G_{i}=\bar{G}_{i}+G_{N_{i}} \Delta N-\beta_{i} \Delta P_{i}-\sum_{j \neq i} \theta_{i j} \Delta P_{j},
$$

where $G_{N_{i}}=\partial G_{i} / \partial N$ is the differential gain, and $\beta_{i}$ $=-\partial G_{i} / \partial P_{i}$ governs nonlinear self-compression of the gain, whereas $\theta_{i j}=-\partial G_{i} / \partial P_{j}$ accounts for the way gain in one mode is affected by the optical power in the other modes. The resulting small-signal rate equations are ${ }^{1}$

$$
\begin{aligned}
& \left(j \Omega+\Gamma_{N}\right) \Delta N=\frac{F_{N}}{V}-\sum_{i} \overline{\bar{G}}_{i} \Delta P_{i}, \\
& \left(j \Omega+\Gamma_{P_{i}}\right) \Delta P_{i}=\frac{\Gamma F_{P_{i}}}{V}+\Gamma G_{N_{i}} P_{i} \Delta N-\sum_{j \neq i} \Gamma \theta_{i j} P_{i} \Delta P_{j},
\end{aligned}
$$

$$
j \Omega \Delta \phi_{i}=-\frac{\alpha_{i}}{2} \Gamma G_{N_{i}} \Delta N+F_{\phi_{i}},
$$

with $\Gamma_{N}=\frac{1}{\tau_{c}}+\Sigma_{i} G_{N_{i}} P_{i}, \Gamma_{P_{i}}=R_{\mathrm{sp}_{i}} / P_{i}+\Gamma \beta_{i} P_{i}$ and $\bar{G}_{i}=\bar{G}_{i}$ $-\beta_{i} P_{i}-\Sigma_{j \neq i} \theta_{j i} P_{j}$. In the above equations $\Gamma$ is the optical confinement factor, $\tau_{c}$ is the differential spontaneous carrier lifetime, $R_{\mathrm{sp}_{i}}$ is the spontaneous emission rate per unit volume into the mode $i$, and $\alpha_{i}$ is the linewidth enhancement factor. The terms $F_{N}, F_{P_{i}}$, and $F_{\phi_{i}}$ are Langevin noise sources associated with carrier recombination and spontaneous emission into mode $i$.

Equations (2)-(4) can be easily solved numerically. However, more insight is gained by making some approximations that are valid in the case of nearly single-mode lasers. The gain margin of the $i$ th mode $\Delta \bar{G}_{i}$, which is defined as the difference between the inverse of the photon lifetime and the gain for that mode, is related to the cw photon density $P_{i}$ according to $\Delta \bar{G}_{i}=R_{\mathrm{sp}_{i}} / P_{i} \cdot{ }^{3}$ For side modes below threshold, the gain margin is large compared to dynamic variations in the gain and dominates the rate at which the photon density fluctuations are damped. Hence, for a side mode, $\Delta P_{i}$ is approximately independent of fluctuations in the carrier density and the photon density of the other modes. From Eq. (3) we get

$$
\Delta P_{i} \cong \frac{\Gamma F_{P_{i}} / V}{j \Omega+R_{\mathrm{sp}_{i}} / P_{i}}, \quad i \neq 0 .
$$

The photon density fluctuations of each of the side modes $\Delta P_{i}$ affect the carrier density and photon density and phase of the main mode. Since the Langevin forces $F_{P_{i}}$ of different modes are uncorrelated, the $\Delta P_{i}$ (for $\left.\neq 0\right)$ can then be considered as independent noise sources in Eqs. (2)-(4) (for $i=0$ ). Using the approach adopted in Ref. 1 we eliminate the correlation between $F_{P_{i}}$ and $F_{N}$ by using $F_{N}=F_{N}^{\prime}$ $-\Sigma_{i} F_{P_{i}}$, where $F_{N}^{\prime}$ is the part of the noise not involving spontaneous emission of photons into one of the longitudinal modes.

The photon and carrier density variations in the main mode are given by

$$
\Delta P_{0}=\Delta P_{0}^{N^{\prime}}+\Delta P_{0}^{P_{0}}+\sum_{i \neq 0} \Delta P_{0}^{P_{i}}
$$




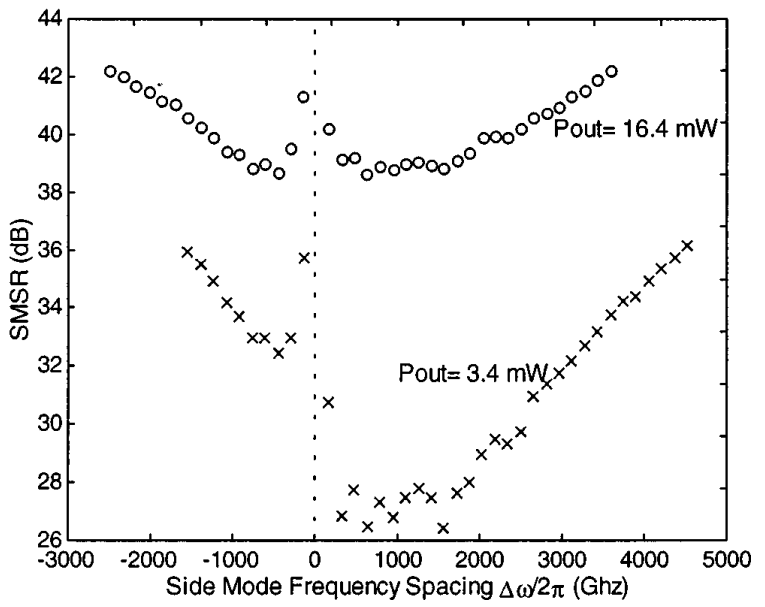

FIG. 1. SMSR at Pout $=3.4 \mathrm{~mW}$ (crosses) and Pout $=16.4 \mathrm{~mW}$ (circles) as a function of the frequency deviation from the main mode.

$$
\Delta N=\Delta N^{N^{\prime}}+\Delta N^{P_{0}}+\sum_{i \neq 0} \Delta N^{P_{i}},
$$

where we use two superscripts to denote the different contributions to the fluctuations $\Delta N, \Delta P_{0}$, and $\Delta \phi_{0}$. The first superscript indicates the physical origin of the noise term, either carrier noise $\left(N^{\prime}\right)$ or spontaneous emission originating photon density noise $(P)$ and phase noise $(\phi)$. In the case of spontaneous emission, a second superscript shows in which mode the spontaneous emission takes place.

Upon substitution of Eq. (5) into the rate equations (2) and (3), the contributions originating from carrier noise, $\Delta P_{0}^{N^{\prime}}$ and $\Delta N^{N^{\prime}}$, and from spontaneous emission in the main mode, $\Delta P_{0}^{P_{0}}$ and $\Delta N^{P_{0}}$, are found to be approximately the same as those obtained with single-mode theory. The additional fluctuations caused by the side modes are given by

$$
\begin{aligned}
\Delta P_{0}^{P_{i}}= & -\left[G_{N_{0}} P_{0} \frac{j \Omega+\Gamma_{P_{i}}+\Gamma \overline{\bar{G}}_{i}}{D(\Omega)}\right. \\
& \left.+\Gamma \theta_{0_{i}} P_{0} \frac{j \Omega+\frac{1}{\tau_{c}}+G_{N_{0}} P_{0}}{D(\Omega)}\right] \Delta P_{i}=p_{0 i} \Delta P_{i}
\end{aligned}
$$

and

$$
\Delta N^{P_{i}}=\frac{1}{\Gamma G_{N_{0}} P_{0}}\left[j \Omega+\Gamma \beta_{0} P_{0}+p_{0 i} P_{0}\right] \Delta P_{0}^{P_{i}},
$$

where the factor $D(\Omega)=-\Omega^{2}+j \gamma_{0} \Omega+\Omega_{0}^{2}$ is the resonant denominator, with $\gamma_{0}$ and $\Omega_{0}$ the damping factor and resonant frequency of the main mode. ${ }^{1}$ These can be well approximated by the values obtained with single-mode theory in the case of a distributed feedback (DFB) laser. From Eqs. (4) and (9), the phase fluctuation in mode $i$ due to spontaneous emission in mode $j$ is given by

$$
\Delta \phi_{i}^{P_{j}}=-\frac{\alpha_{i}}{2} \frac{G_{N_{i}}}{j \Omega} \Gamma \Delta N^{P_{j}}
$$

Equation (8) shows the additional fluctuation in the photon density of the main mode, which is anticorrelated with the noise source $\Delta P_{i}$ that originates it. Two physical effects Downloaded 31 Aug 2006 to 131.215.240.9. Redistribution subject

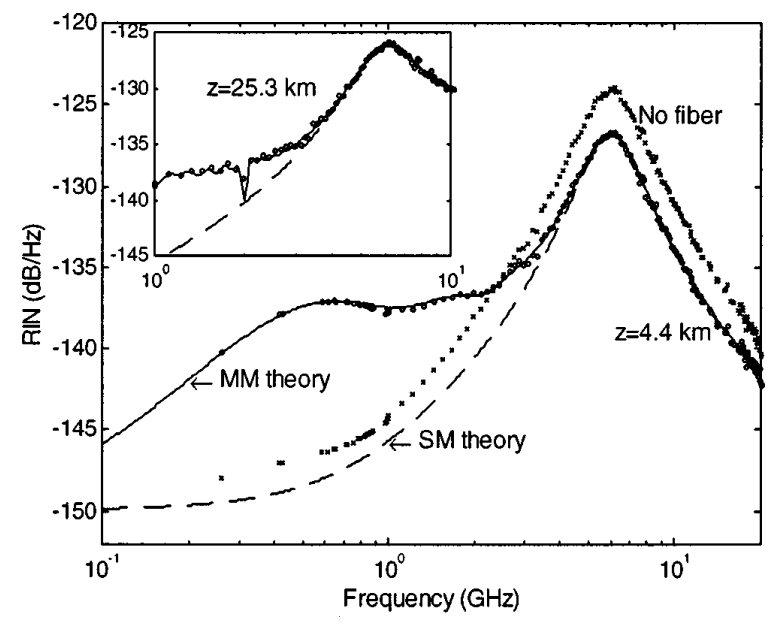

FIG. 2. RIN before fiber and after 4.4 and $25.3 \mathrm{~km}$ (inset) of fiber at Pout $=3.4 \mathrm{~mW}$, Ibias $=1.6 \mathrm{Ith}, \mathrm{SMSR}=27 \mathrm{~dB}$. The dots are experimental, the solid line is multimode theory, and the dashed line is single-mode theory.

are present: first, depletion of carriers due to spontaneous and stimulated emission into the $i$ th side mode, and second, compression of the gain of the main mode by the power in the side modes.

The effect of the side modes is only important at frequencies well below the relaxation resonance frequency of the main mode. Closer to resonance $\Delta P_{0}^{P_{i}}$ dominates over $\Delta P_{0}^{P_{i}}$ and $\Delta P_{i}$, and beyond resonance the side-mode fluctuation is highly damped due to its low-pass characteristic, as seen in Eq. (5). At frequencies below $1 / \tau_{c}$ and $\bar{G}_{0}$, the inverse of the response time of carriers and photons, respectively, the carrier and photon density are adiabatically related, and since in this regime the carrier density is clamped, $\Delta N$ is practically zero. Considering the effect of $\Delta P_{i}$ separately, spontaneous emission into the side-mode $i$ tends to decrease the carrier density, as does stimulated emission into the side mode and the main mode. Hence, the condition $F_{P_{i}} / V+\overline{\bar{G}}_{i} \Delta P_{i}+\overline{\bar{G}}_{0} \Delta P_{0}^{P_{i}} \approx 0$ has to be satisfied. This relation can also be easily derived from the rate equations (2)(4) if we ignore gain compression. Unless the side mode is so weak that the number of photons in the side mode is comparable to the number of photons originated by spontaneous emission, the factor corresponding to the stimulated gain dominates. Taking $\bar{G}_{i} \approx \bar{G}_{0}$, which is a valid approximation for the dominant side modes since the gain spectrum of semiconductor lasers is quite broad $(\sim 50 \mathrm{~nm})$, we end up with $\Delta P_{0}^{P_{i}} \sim-\Delta P_{i}$. This negative correlation between the noise caused by $F_{P_{i}}$ in the main mode and the side mode is what causes cancellation of the mode partition noise at the output of the laser when we compute the total RIN.

For a multimode laser, the total detected intensity is the sum of the intensity in each mode. Since the different noise sources are uncorrelated, the spectral densities of the fluctuations are additive, and therefore, we can consider their effects individually. The total RIN at the receiver in the case of a nearly single-mode laser will then be given by

$$
\operatorname{RIN}(\Omega, z) \approx \operatorname{RIN}_{0}(\Omega, z)+\sum_{i \neq 0} \frac{\left\langle\left|H_{0}^{P_{i}} \Delta P_{0}^{P_{i}}+H_{i}^{P_{i}} \Delta P_{i}\right|^{2}\right\rangle}{P^{2}},
$$


where RIN $_{0}$ is the RIN obtained with single-mode theory in Ref. 1 , and the second term is the additional noise that comes from the side modes. We have used the fact that the magnitude of fluctuations caused by the conversion between the frequency chirp (FM) and the intensity modulation (AM) is weighted by the power in the mode [see Eq. (12) below]. Consequently, the phase noise in the side modes, $F_{\phi_{i}}$, can be neglected. In Eq. (11), $H_{i}^{P_{i}}(\Omega)$ is the small-signal transfer function for propagation of intensity variations of the mode $i$ driven by $F_{P_{j}}$. This transfer function is a generalization of $H_{2}(\Omega)$ in Ref. 1, which corresponds to $H_{0}^{P_{0}}(\Omega)$ in the present notation, for the multimode case. In the case of propagation through dispersive optical fiber $H_{i}^{P_{i}}(\Omega)$ is found to be

$$
H_{i}^{P_{i}}(\Omega)=\left[\cos (\theta)-2 P_{i} \frac{\Delta \phi_{i}^{P_{j}}}{\Delta P_{i}^{P_{j}}} \sin (\theta)\right] \exp \left(j \vartheta_{i}\right),
$$

where $\theta=-1 / 2 \beta_{2} \Omega^{2} z$ and $\vartheta_{i}=-\beta_{2} \Delta \omega_{i} \Omega z$, with $\beta_{2}$ the fiber group velocity dispersion coefficient, $z$ the fiber length, and $\Delta \omega_{i}$ the optical frequency spacing between mode $i$ and the main mode. The factor $\exp \left(j \vartheta_{i}\right)$ accounts for the group delay of side modes relative to the main mode.

Substitution of Eqs. (9) and (10) into Eq. (12) yields

$$
\begin{aligned}
& H_{0}^{P_{i}} \Delta P_{0}^{P_{i}}+H_{i}^{P_{i}} \Delta P_{i} \\
&=\left[\cos (\theta)\left(\exp \left(j \vartheta_{i}\right)+p_{0 i}\right)+\alpha_{0}\right. \\
&=\sin (\theta)\left(p_{0 i}+\frac{\Gamma\left(p_{0 i} \beta_{0}+\theta_{0 i}\right) P_{0}}{j \Omega}\right) \\
&\left.\quad \times\left(1+\frac{\alpha_{j}}{\alpha_{0}} \frac{G_{N_{j}} P_{j}}{G_{N_{0}} P_{0}} \exp \left(j \vartheta_{i}\right)\right)\right] \Delta P_{i} \\
& \approx {\left[\cos (\theta)\left(\exp \left(j \vartheta_{i}\right)+p_{0 i}\right)+p_{0 i} \alpha_{0} \sin (\theta)\right] \Delta P_{i} . }
\end{aligned}
$$

Equation (13) explains why even highly suppressed side modes can contribute significantly to the RIN after propagation in dispersive fiber. As a consequence of the difference in group velocities of the longitudinal modes in dispersive fiber, fluctuations originating in the side modes no longer cancel out after propagation, but instead cause oscillations in the noise power at the receiver. It can be seen that there is an overall increase in the RIN that rolls off at high frequencies due to the low-pass characteristic of the noise source $\Delta P_{i}$. The last approximation in Eq. (13) is valid for $|\theta| \ll 1$, that is, for small propagation distance and moderate frequencies. As in the single-mode case, ${ }^{1}$ excess noise for side modes is reduced after propagation when $\alpha \beta_{2}>0$.

In order that MPN have a significant contribution at a given modulation frequency, $\vartheta_{i}$ has to be close to $\pi(1$ $+2 m$ ) for one or several of the dominant side modes, where $m$ is an integer. The frequency separation between the main mode and the $i$ th mode is given approximately by $\left|\Delta \omega_{i}\right|$ $\sim|i| 2 \pi c / L$, with $L$ the laser length, $c$ the speed of light, and $i$ the mode index. Thus, assuming a typical value $\beta_{2}$ $=-20 \mathrm{ps}^{2} / \mathrm{km}$ at $1.55 \mu \mathrm{m}$, the condition above becomes $\Omega \approx[(1+2 m) L / z] /|i| 2 c\left|\beta_{2}\right| \approx 0.1[(1+2 m) /|i|] L(\mu \mathrm{m}) / z(\mathrm{~km})$. If the length of the laser in $\mu \mathrm{m}$ divided by the mode number, Downloaded 31 Aug 2006 to 131.215.240.9. Redistribution subject

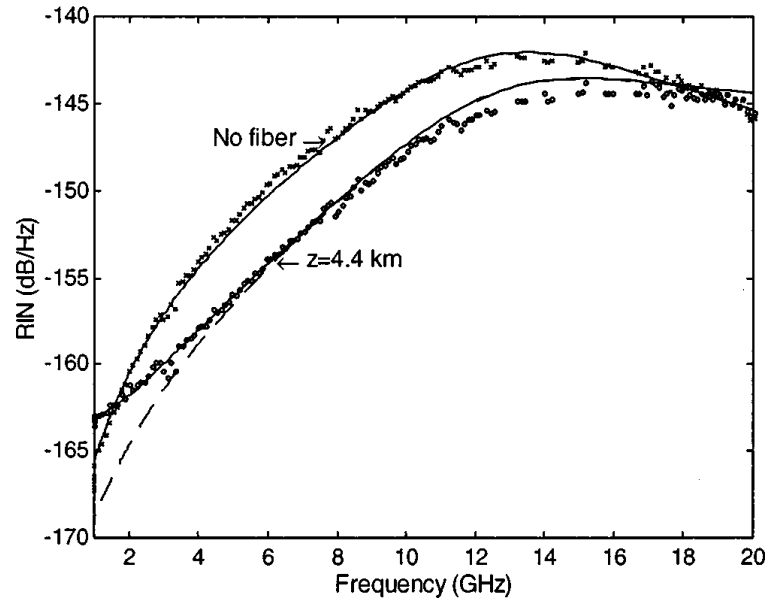

FIG. 3. RIN after $4.4 \mathrm{~km}$ of fiber at Pout $=16.4 \mathrm{~mW}$, Ibias $=4.1 \mathrm{Ith}$, SMSR $=40 \mathrm{~dB}$. Same notation as in Fig. 2 .

$L(\mu \mathrm{m}) /|i|$, is much larger than the propagation distance $z$ in $\mathrm{km}$, the frequencies at which the previous condition is satisfied are so high that an increase in RIN is no longer seen. These conditions hold for short lengths of fiber and side modes close to the main mode.

The relative intensity noise of a $250 \mu \mathrm{m}$ length DFB laser at $1.54 \mu \mathrm{m}$ was measured before and after propagation in various lengths of standard single-mode optical fiber (see Figs. 1-3). A good fit to the standard single-mode theory was obtained before adding fiber. However, an increase in the RIN over the frequency range of $500 \mathrm{MHz}$ to $5 \mathrm{GHz}$ depending on fiber length and laser output power was observed when RIN after propagation was measured. Although the side modes in the stop band of the DFB laser are highly suppressed, there are many FP cavity modes that have similar power over a large bandwidth (see Fig. 1). Including only the strongest side mode in the calculations, the theory does not predict what is experimentally observed.

We measured the optical spectrum to determine the power in $\sim 40$ side modes together with their separation $\Delta \omega_{i}$ from the main mode. The measured RIN without fiber was used to determine $R_{\mathrm{sp}_{0}}, \Omega_{0}$, and $\gamma_{0}$ at each operation point. The rest of the parameters were estimated from the RIN with fiber so as to obtain the best fits, yielding $\alpha=-4.6, \tau_{c}$ $=0.27 \mathrm{~ns}, \tau_{\mathrm{ph}}=5.5 \mathrm{ps}$, and $\beta_{2}=-20 \mathrm{ps}^{2} / \mathrm{km}$.

For some fiber lengths the single-mode theory predicts a reduction in RIN, ${ }^{1}$ which is not necessarily achieved experimentally due to these multimode effects. In the inset of Fig. 2 , the notch corresponds to the condition that $1 / 2 \beta_{2} \Delta \omega \Omega z$ is an integer multiple of $\pi$, with $\Delta \omega$ the mean-mode spacing, at which point all modes arrive at the detector in phase. The effect of MPN is observed even at high output powers and SMSR's (Fig. 3).

The authors are very grateful to Ortel Corporation. This work was supported by DARPA, ONR, and AFSR.

${ }^{1}$ W. K. Marshall, J. Paslaski, and A. Yariv, Appl. Phys. Lett. 68, 2496 (1996).

${ }^{2}$ R. H. Wentworth, G. E. Bodeep, and T. E. Darcie, J. Lightwave Technol. 10, 84 (1992).

${ }^{3}$ K. Petermann, Laser Modulation and Noise (Kluwer, Boston, 1991) 